\title{
Germination and salinity tolerance of seeds of sixteen Fabaceae species in Thailand for reclamation of salt-affected lands
}

\author{
YONGKRIAT KU-OR ${ }^{1}$, NISA LEKSUNGNOEN ${ }^{1,2, \boldsymbol{}}$, DAMRONGVUDHI ONWIMON ${ }^{3}$, PEERAPAT DOOMNIL ${ }^{1}$ \\ ${ }^{1}$ Department of Forest Biology, Faculty of Forestry, Kasetsart University. 50 Phahonyothin Rd, Lat yao, Chatuchak, Bangkok 10900, Thailand \\ ${ }^{2}$ Center for Advanced Studies in Tropical Natural Resources, National Research University, Kasetsart University. 50 Phahonyothin Rd, Lat yao, \\ Chatuchak, Bangkok 10900, Thailand. "email: ffornsl@ku.ac.th \\ ${ }^{3}$ Department of Agronomy, Faculty of Agriculture, Kasetsart University. 50 Phahonyothin Rd, Lat Yao, Chatuchak, Bangkok 10900, Thailand.
}

Manuscript received: 26 March 2020. Revision accepted: 24 April 2020.

\begin{abstract}
Ku-Or Y, Leksungnoen N, Onwinom D, Doomnil P. 2020. Germination and salinity tolerance of seeds of sixteen Fabaceae species in Thailand for reclamation of salt-affected lands. Biodiversitas 21: 2188-2200. Over the years, areas affected by salinity have increased dramatically in Thailand, resulting in an urgent need for reclamation of salt-affected areas using salinity tolerant plant species. In this context, seed germination is an important process in plant reproduction and dispersion. This research aimed to study the ability of 16 fabaceous species to germinate and tolerate salt concentrations of at 6 different levels (concentration of sodium chloride solution, i.e., $0,8,16,24,32$, and $40 \mathrm{dS} \mathrm{m}^{-1}$ ). The germination test was conducted daily for 30 days, and parameters such as germination percentage, germination speed, and germination synchrony were calculated. The electrical conductivity (EC50) was used to compare the salt-tolerant ability among the 16 species. Our results showed that the germination percentage, germination speed, and germination synchrony of all species decreased with an increase in salinity concentration. The mean germination time increased from 2-4 days under $0 \mathrm{dS}^{-1}$ to $4-10$ days under $16 \mathrm{dS} \mathrm{m}^{-1}$, indicating that the seeds germinated at a slower rate as the salinity levels increased. Seed germination percentage was more than $80 \%$ across all the species at salinity levels between 0 to $16 \mathrm{dS} \mathrm{m}^{-1}$. Sesbania grandiflora, Senna siamea, and Dalbergia cochinchinensis had a high $\mathrm{EC}_{50}$ value of $33.56,32.93$, and $30.83 \mathrm{dS} \mathrm{m}^{-1}$ respectively, suggesting that these species were the three most salt-tolerant species in this study. As such, the establishment of these species in salt-affected areas should be studied further in order to reclaim such affected lands.
\end{abstract}

Keywords: EC50, Fabaceae, germination, morphology, salinity

\section{INTRODUCTION}

Soil salinity is a big problem in every part of the world. It is estimated that the land area under salinity is approximately 955 million ha or $\% 15$ of the total land area of the world (Metternicht and Zinck 2008). In Thailand, inland salinity affects approximately 3.15 million ha, with reported annual average levels of surface salinity between 40-45 $\mathrm{dS} \mathrm{m}^{-1}$ (Leksungnoen 2017). Most of the salt-affected areas are found in the northeastern part of Thailand, while the central plains and coastal areas are affected by the salt-sprayed from oceanic waters or tidal bores (Kheoruenromne 2007; Jedrum et al. 2014). Saltaffected soil causes a reduction in agricultural productivity and can lead to abandonment of the land as not many plants can survive in such soils. However, there are ways to reduce the extent of this problem, such as draining the soil with fresh water to wash the salt crust formed on the surface (Egamberdieva and Mamedov 2015). However, this method is expensive and not sustainable.

Another sustainable method is planting deep-rooted pioneer species in salt-affected areas, but the establishment can take a long time. Plants with deep roots can utilize the saltwater and prevent upwelling of salt to the soil surface. As a result, agricultural crops, which have shallow root systems, have a better chance to grow. This method can be used to reduce or mitigate soil salinity issues in the long term. Munns and Tester (2008) reported that droughttolerant and salt-tolerant plants have similar responses as such stresses result from low water potential. As a result, the roots of plants find it difficult to use water. Therefore, plants under salt-related stress show signs of physiological dehydration similar to those under drought-related stress. Thus, trees in dry areas such as in dipterocarp forests would have a tendency to be salt-tolerant (Suntisuk 2006; Chanrattana et al. 2013; Leksungnoen et al. 2014).

The Fabaceae family consists of more than 20,000 species, making it the third-largest flowering plant family after Asteraceae and Orchidaceae (Azani et al. 2017). The Fabaceae species are mostly found in dry dipterocarp forests in Thailand, where drought can last for up to 4-7 months per year. Drought-tolerant species growing in dry deciduous forest mostly belong to the Fabaceae family, such as Albizia lebbeck, Albizia odoratissima, Bauhinia saccocalyx, Butea monosperma, Dalbergia cultrate, Dalbergia nigrescens, Dalbergia oliveri, Peltophorum dasyrachis, Pterocarpus macrocarpus, Sindora siamensis, and Xylia xylocarpa (Santisuk 2006), which may have a good potential for salt tolerance. Fabaceous species are very important to people in Thailand because they provide for various human needs including food (derived from leaves, fruits, and seeds), shelter (derived from timber), clothing (derived from fiber), and medicine such as root and bark of Peltophorum africanum, which are used to treat 
diarrhea, dysentery, sore throat, wounds, back, and joint pains (Chikoto et al. 2005).

Seedling establishment is a key step in determining plant growth and development in salt-affected areas. Seeds require various mechanisms to germinate in a salt-stress environment, or else they will remain in the quiescent stage (Moud and Maghsoudi 2008). Salinity adversely affects early seed germination and growth of seedling (Nawaz et al. 2012). High salinity level results in sodium toxicity that can destroy the cells of a seed (Panuccio et al., 2014; Izadi et al. 2014; Pessarakli 2015; Pakar et al. 2016). The degree of specific ion toxicity, or salt tolerance, varies with its genetics, also at different developmental stages (Ahmad et al. 2013; Bueno et al. 2017). Ku-or and Leksungnoen (2018) reported that Leucaena leucocephala, Albizia lebbeck, and Buchanania siamensis could germinate in saline soils $\left(40 \mathrm{dS} \mathrm{m}^{-1}\right)$ and were classified as highly salttolerant seeds.

Germination process is important during the reproduction and dispersion of a plant community (ISTA 2017). Seed germination process can be divided into 3 phases. The first phase is the water absorption phase (imbibition), the second is the digestion of food by enzymes in a seed (lag phase), and the third is the growth of radicle (radicle protrusion) (Hubbard et al. 2012; Bewley and Black 2012; Baskin and Baskin 2014; Han and Yang 2015). Imbibition is an essential prerequisite for germination and its rate is governed by the surrounding soil water potential and the resistance to the movement of water in a soil-seed system (Linkies et al. 2010; Weitbrecht et al. 2011).

For natural reproduction in salt-affected areas, it is necessary to study and identify species that produce seeds that can tolerate high salinity conditions. The objective of this research was to study the ability of seeds selected from species to germinate under different concentration levels of Sodium chloride $(\mathrm{NaCl})$ solution, i.e., $0,8,16,24,32$, and $40 \mathrm{dS} \mathrm{m} \mathrm{m}^{-1}$. This was done for the seeds of 16 species of Fabaceae family in Thailand, i.e., Adenanthera pavonina,
Albizia saman, Albizia procera, Cassia bakeriana, Cassia fistula, Cassia javanica, Dalbergia cochinchinensis, Dalbergia oliveri, Delonix regia, Millettia leucantha, Peltophorum dasyrrhachis, Peltophorum pterocarpum, Pterocarpus macrocarpus, Senna siamea, Senna surattensis, and Sesbania grandiflora.

\section{MATERIALS AND METHODS}

\section{Seed samples}

Seeds of the 16 species in the Fabaceae family were collected during the years 2018 and 2019 from various locations in Thailand, as listed in Table 1 and Figure 1. Most species (12 out of 16 species) were obtained from the Royal Forest Department. The remaining 4 species were collected from at least 5-7 mature and healthy mother plants to ensure a good seed source. The selected species are native to the deciduous forest or open areas which are likely to be drought-tolerant (Munns 2002).

\section{Seed morphological analysis}

Different seed sizes can result in varying germination rates according to differences in the nutrients accumulated in each seed. In order to measure the size, width, and length, 100 seeds from each species were randomly measured using a Vernier Caliper (Brand AND Model AD5763-150, A\&D Company, Limited, Japan). The average seed size was then compared using ANOVA test.

The shape and color of the seeds were recorded and photographed using a stereomicroscope at $40 \times$ magnification (Brand Zeiss Model Stemi 508 doc, Carl Zeiss Ltd., Gottingen, Germany).

The seed weight was measured by weighing 100 random seeds from each species. Four replications were measured with an analytical balance having a resolution of $0.001 \mathrm{~g}$ (Precisa Model XT 620M).

Table 1. Sources of seeds used in this study

\begin{tabular}{|c|c|c|c|c|}
\hline No. & Scientific name & Sub-family & Latitude/longitude & Year \\
\hline 1 & Adenanthera pavonina & Caesalpinioideae & $13^{\circ} 45^{\prime} 22.7916^{\prime \prime} \mathrm{N} ; 100^{\circ} 30^{\prime} 6.3432^{\prime \prime} \mathrm{E}$ & 2019 \\
\hline 2 & Albizia saman & Caesalpinioideae & $9^{\circ} 7^{\prime} 54.444 " \mathrm{~N} ; 99^{\circ} 20^{\prime} 1.0248^{\prime \prime} \mathrm{E}$ & 2019 \\
\hline 3 & Albizia procera & Caesalpinioideae & $16^{\circ} 49^{\prime} 16.0428^{\prime \prime} \mathrm{N} ; 100^{\circ} 15^{\prime} 57.0744 " \mathrm{E}$ & 2019 \\
\hline 4 & Cassia bakeriana & Caesalpinioideae & $16^{\circ} 25^{\prime} 55.8984 " \mathrm{~N} ; 102^{\circ} 49^{\prime} 25.0464 " \mathrm{E}$ & 2018 \\
\hline 5 & Cassia fistula & Caesalpinioideae & $14^{\circ} 58^{\prime} 20.568^{\prime \prime} \mathrm{N} ; 102^{\circ} 5^{\prime} 57.8508^{\prime \prime} \mathrm{E}$ & 2018 \\
\hline 6 & Cassia javanica & Caesalpinioideae & $19^{\circ} 54^{\prime} 41.8716^{\prime \prime} \mathrm{N} ; 99^{\circ} 49^{\prime} 54.516^{\prime \prime} \mathrm{E}$ & 2018 \\
\hline 7 & Delonix regia & Caesalpinioideae & $13^{\circ} 6^{\prime} 39.924^{\prime \prime} \mathrm{N} ; 9^{\circ} 56^{\prime} 41.1756^{\prime \prime} \mathrm{E}$ & 2019 \\
\hline 8 & Peltophorum dasyrrhachis & Caesalpinioideae & $16^{\circ} 3^{\prime} 10.4076^{\prime \prime} \mathrm{N} ; 103^{\circ} 39^{\prime} 11.7324^{\prime \prime} \mathrm{E}$ & 2018 \\
\hline 9 & Peltophorum pterocarpum & Caesalpinioideae & $16^{\circ} 29^{\prime} 6.504^{\prime \prime} \mathrm{N} ; 99^{\circ} 31^{\prime} 28.8516^{\prime \prime} \mathrm{E}$ & 2018 \\
\hline 10 & Senna siamea & Caesalpinioideae & $16^{\circ} 25^{\prime} 55.8984 " \mathrm{~N} ; 102^{\circ} 49^{\prime} 25.0464 " \mathrm{E}$ & 2018 \\
\hline 11 & Senna surattensis & Caesalpinioideae & $19^{\circ} 11^{\prime} 29.5764^{\prime \prime} \mathrm{N} ; 99^{\circ} 52^{\prime} 47.8524^{\prime \prime} \mathrm{E}$ & 2018 \\
\hline 12 & Dalbergia cochinchinensis & Faboideae & $16^{\circ} 25^{\prime} 55.8984 " \mathrm{~N} ; 102^{\circ} 49^{\prime} 25.0464 " \mathrm{E}$ & 2019 \\
\hline 13 & Dalbergia oliveri & Faboideae & $16^{\circ} 3^{\prime} 10.4076^{\prime \prime} \mathrm{N} ; 103^{\circ} 39^{\prime} 11.7324^{\prime \prime} \mathrm{E}$ & 2019 \\
\hline 14 & Millettia leucantha & Faboideae & $16^{\circ} 29^{\prime} 6.504^{\prime \prime} \mathrm{N} ; 99^{\circ} 31^{\prime} 28.8516^{\prime \prime} \mathrm{E}$ & 2019 \\
\hline 15 & Pterocarpus macrocarpus & Faboideae & $16^{\circ} 3^{\prime} 10.4076^{\prime \prime} \mathrm{N} ; 103^{\circ} 39^{\prime} 11.7324^{\prime \prime} \mathrm{E}$ & 2019 \\
\hline 16 & Sesbania grandiflora & Faboideae & $13^{\circ} 66^{\prime} 39.924^{\prime \prime} \mathrm{N} ; 99^{\circ} 56^{\prime} 41.1756^{\prime \prime} \mathrm{E}$ & 2019 \\
\hline
\end{tabular}




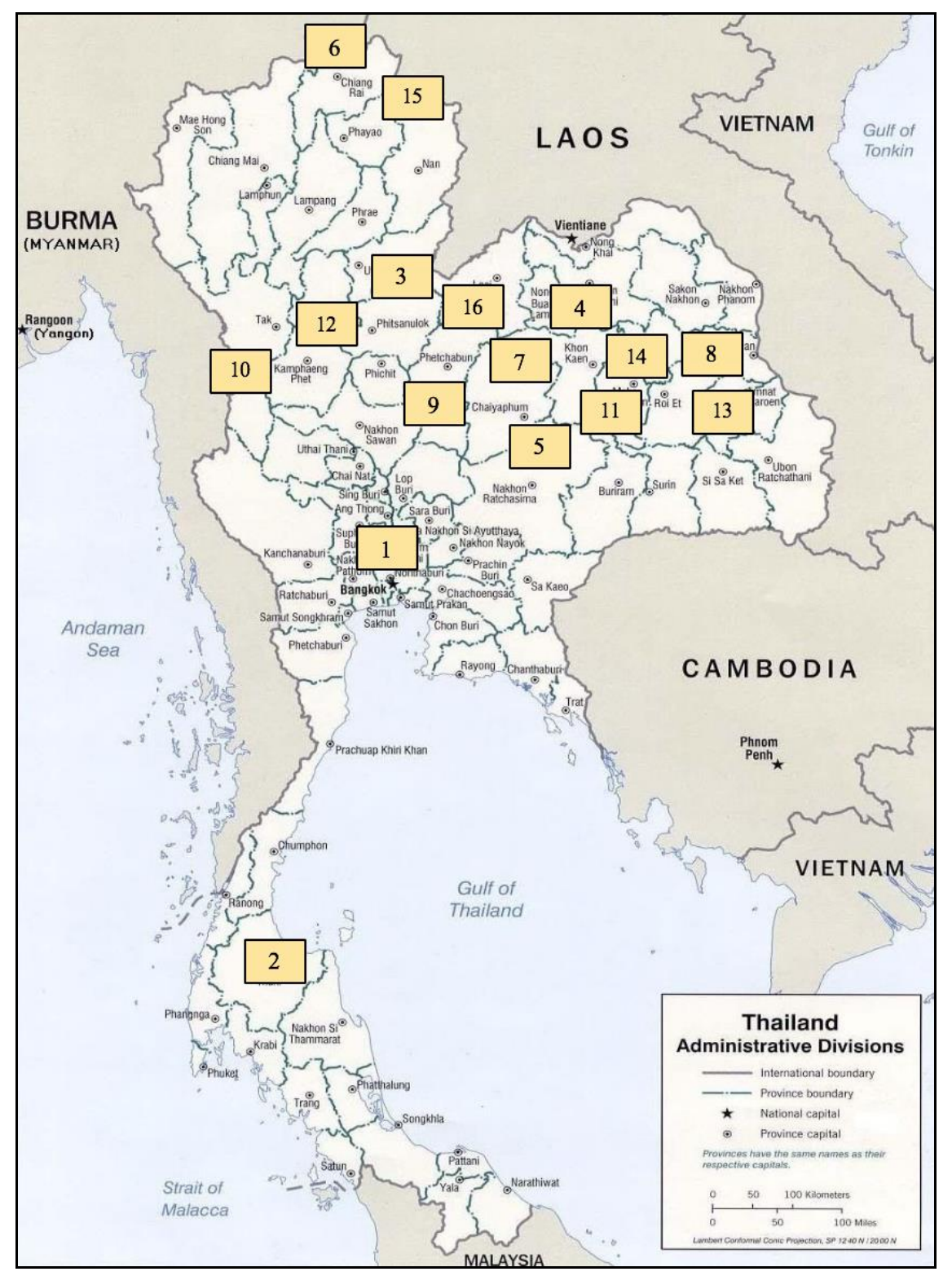

Figure 1. Location of seed collection sites in Thailand. Number location refers to Table 1.

\section{Seed moisture content determination}

Seed moisture content (SMC) was measured by the high constant temperature oven method (ISTA 2017). At least 25 fresh seeds from each species were weighed individually on an analytical balance with a resolution of $0.001 \mathrm{~g}$ to obtain an initial weight. The seed sample was then oven-dried at a temperature of $103 \pm 3{ }^{\circ} \mathrm{C}$ for $17 \pm 1$ hours or until a constant mass was obtained. The sample was weighed again immediately to obtain the oven-dried weight. Four replicates were measured for each species.

\section{Evaluation of seed salinity tolerance}

\section{Preparation and treatment of the seed}

According to a report from The Royal Forest Department, fabaceous seeds have to be primed before a germination test. Water at a temperature of $100{ }^{\circ} \mathrm{C}$ was poured on the seeds to soak them for 20 minutes. The seeds were then continuously soaked in water at $25^{\circ} \mathrm{C}$ for 24 hours before sowing in the testing boxes (size $114 \mathrm{~mm}$ $\times 114 \mathrm{~mm} \times 65 \mathrm{~mm}$ ).

Different concentrations of $\mathrm{NaCl}$ solution were prepared and the target concentration was determined using an electrical conductivity meter (DIGICON, CD-437SD, Digicon Company, Germany) (Table 2). Twenty $\mathrm{ml}$ of $\mathrm{NaCl}$ solution was added per box for each concentration level with 4 replicates for each combination of concentration and species. In this study, the salinity levels were increased in steps of $8 \mathrm{dS} \mathrm{m}^{-1}$, given the plant response to salinity proposed by Arunin (1996). The maximum concentration was set at $40 \mathrm{dS} \mathrm{m}^{-1}$, as this is the average soil concentration measured in the salt-affected areas of northeastern Thailand (Leksungnoen 2017). 
Table 2. Preparation of $\mathrm{NaCl}$ solution at various concentration levels

\begin{tabular}{llll}
\hline $\begin{array}{c}\text { Salt conc. level } \\
\left(\mathbf{d S ~ m}^{-1}\right)\end{array}$ & $\begin{array}{c}\text { NaCl salt } \\
\text { content } \mathbf{( g )}\end{array}$ & $\begin{array}{c}\text { Deionized } \\
\text { water }(\mathbf{g})\end{array}$ & $\begin{array}{c}\text { Osmotic } \\
\text { potential } \\
(\mathbf{M P a})\end{array}$ \\
\hline 0 & 0.00 & 1000.00 & 0 \\
8 & 5.12 & 994.88 & -0.288 \\
16 & 10.24 & 989.76 & -0.576 \\
24 & 15.36 & 984.64 & -0.864 \\
32 & 20.48 & 979.52 & -1.152 \\
40 & 25.60 & 974.40 & -1.440 \\
\hline
\end{tabular}

All germination boxes were stored in a growth chamber and were controlled at a temperature and humidity of $25 \pm$ $3{ }^{\circ} \mathrm{C}$ and $80-90 \%$, respectively, in the absence of light, throughout the experiment. Germination was measured daily for 30 days. Seeds were considered to have germinated when the roots had protruded and could be seen by the naked eye. The mean germination time, germination speed, and germination synchrony were calculated as follow (Aravind et al. 2019);

\section{Mean germination time (MGT)}

Mean germination time (MGT) is the average length of time required for maximum germination of a seed lot and is estimated according to the following formula.

$$
\operatorname{MGT}=\frac{\underset{\mathrm{i}=1}{\mathrm{k}} \mathrm{N}_{\mathrm{i}} \mathrm{T}_{\mathrm{i}}}{\mathrm{i}_{\mathrm{i}=1} \mathrm{~N}_{\mathrm{i}}},
$$

Where; $T_{i}$ is the time from the start of an experiment to the $i^{\text {th }}$ day, $\mathrm{N}_{\mathrm{i}}$ is the number of seeds germinated at $i^{\text {th }}$ day (not the accumulated number, but the number corresponding to the $i^{\text {th }}$ day), and $\mathrm{k}$ is the last day of germination (30 days in this experiment).

\section{Germination speed (GS)}

Germination speed (GS) is the rate of germination in terms of the total number of seeds that germinate in a given time interval. It was calculated as follows:

$$
\mathrm{GS}=\frac{\mathrm{N}_{1}}{\mathrm{~T}_{1}}+\frac{\mathrm{N}_{2}}{\mathrm{~T}_{2}}+\frac{\mathrm{N}_{3}}{\mathrm{~T}_{3}}+\ldots+\frac{\mathrm{N}_{\mathrm{n}}}{\mathrm{T}_{\mathrm{n}}},
$$

Where: $\mathrm{N}_{1}, \mathrm{~N}_{2}, \mathrm{~N}_{3}, \cdot \cdot \cdot, \mathrm{N}_{\mathrm{n}}$ are the number of germinated seeds observed each day and $T_{1}, T_{2}, T_{3}, \cdots, T_{n}$ are the days after germinating.

\section{Germination synchrony (Z)}

Germination synchrony represents the uniformity of germination. It was calculated as follows:

$$
\mathrm{Z}=\frac{\sum_{i=1}^{k} \mathrm{C}_{\mathrm{N}_{\mathrm{i}}, 2}}{\mathrm{C}_{\Sigma \mathrm{N}_{\mathrm{i}}, 2}}
$$

Where: $\mathrm{C}_{\mathrm{Ni}}, 2$ is the partial combination of two germinated seeds from among $\mathrm{N}_{\mathrm{i}}$ (the number of seeds germinated on the $i^{\text {th }}$ day) during the $\mathrm{i}^{\text {th }}$ day interval and $\mathrm{C}_{\mathrm{N}_{\mathrm{i}}, 2}$ is the partial combination of the two germinated seeds from among the total number of seeds germinated in the final count, assuming that all the seeds that germinated did so simultaneously.

\section{Electrical conductivity causing the germination percentage to decrease by $50 \%\left(\mathrm{EC}_{50}\right)$}

$\mathrm{EC}_{50}$ is the value of electrical conductivity that causes the germination to decrease by $50 \%$ and is used to compare the salinity tolerance capacity of a species. The calculation is based on the percentage of germinated seeds at a given concentration level. The correlation between cumulative germination and salt concentration was analyzed using the $\mathrm{R}$ program. The total germination at each concentration level was normalized by comparing with the control (0 dS $\mathrm{m}^{-1}$ ). Then, a simple linear correlation between the normalized germination and electrical conductivity was calculated. The best fit curve was determined as indicated by the $\mathrm{R}^{2}$ value and the equation was used to solve for the value of $\mathrm{EC}_{50}$.

\section{Statistical analysis}

This experiment was designed as a two-way factorial with 2 main factors: (i) species from which the seed was produced (16 species); and (ii) $\mathrm{NaCl}$ concentration (6 levels); each treatment had 4 replicates. The $\mathrm{R}$ program was used for the analysis of variance (ANOVA) with paired comparisons using the Least Square Difference (LSD) method at a significance level of $P<0.05$. In order to compare the salinity tolerance capacity among the species, $\mathrm{EC}_{50}$ was used to classify and compare the salt tolerance among the 16 species.

\section{RESULTS AND DISCUSSION}

The seeds of 16 fabaceous species tested in this study were mostly round or elliptical in shape (Table 3). Only the seeds of $D$. cochinchinensis and $D$. oliveri were of kidney shape. The colors of seed coat were mostly yellow to brown or brown to black (Figure 2), while the seeds of $A$. pavonina were the only ones that were red. Seed coat of all species was smooth and glossy. Seed size varied among species with a length between 4.65 to $23.82 \mathrm{~mm}$ and width between 2.47 to $14.67 \mathrm{~mm}$. Among all the species, the seeds of $M$. leucantha were the largest in size while $S$. grandiflora seeds were the smallest. Seed weight was correlated with its size. The heaviest seed was $M$. leucantha while $S$. siamea, S. surattensis and S. grandiflora were relatively lighter than others (Table 3). Seed moisture was approximately 7 to $16 \%$ with the seeds of $D$. oliveri having the highest SMC of $16.01 \pm 0.21 \%$ while $P$. pterocarpum seeds had the lowest SMC $(7.07 \pm 0.27 \%)$. Seed moisture was not correlated with the seed size. All seeds in this study were classified as having orthodox behavior. An orthodox seed can survive drying and/or freezing during ex-situ conservation and is considered to have a longer shelf life. Such kinds of seeds can be stored at lower temperatures for a long time. 
The seeds of all the species in this study showed an epigeal germination pattern in which the hypocotyl grows elongated above the soil surface. The total germination percentage of seeds was between $0-95 \%$. This number decreased with increasing salinity concentration (Figure 3 ). Based on the average germination percentage under all the salinity concentrations $\left(0-40 \mathrm{dS} \mathrm{m} \mathrm{m}^{-1}\right)$, the seeds can be classified into 4 groups, namely: (i) high germination percentage ( $>65 \%$ ), which included S. grandiflora, $P$. dasyrrhachis, and $P$. pterocarpum; (ii) moderate germination percentage (45-65\%), which included $S$. surattensis, A. procera, S. siamea, and P. macrocarpus; (iii) low germination percentage (15-45\%), which included D. cochinchinensis, A. saman, D. regia, and M. leucantha; and 4) the lowest germination percentage ( $<15 \%)$, which included C. fistula, C. bakeriana, C. javanica, A. pavonina, and $D$. oliveri.

Under control treatment $\left(0 \mathrm{dS} \mathrm{m}^{-1}\right), P$. dasyrrhachis seeds had the highest germination percentage of $91 \%$, while $D$. oliveri seed had the lowest germination percentage at $2 \%$ (Figure 3 ). The seeds of all the species were able to germinate well $(40-80 \%)$ at salinity levels between $8-16 \mathrm{dS} \mathrm{m}^{-1}$ with no statistically significant difference among the species (Figure 3). Salinity between 8-16 dS m$~ m^{-1}$ is considered as high in which only salt tolerant species can grow (Arunin 1989). At these levels, $P$. dasyrrhachis, $P$. ptercarpum, $S$. grandiflora, and $S$. surattensis seeds had the highest germination percentage. In summary, such seeds can germinate naturally on the soil surface that has salinity levels lower than $16 \mathrm{dS} \mathrm{m}^{-1}$, without any decrease in germination percentage.

Salinity concentration higher than $16 \mathrm{dS} \mathrm{m}^{-1}$ is considered as highly saline in which only salt-tolerant species can thrive while most species cannot survive. We observed the germination of seed under high and extremely high salinity concentration (24-40 $\mathrm{dS} \mathrm{m}^{-1}$ ). Under salinity level of $24 \mathrm{dS} \mathrm{m}^{-1}$, a significant reduction in germination was observed in most seeds with only $S$. grandiflora seeds having high germination of $83 \%$ (Figure 3). In addition, $S$. siamea and $A$. procera also had germination of more than $50 \%$. At $32 \mathrm{dS} \mathrm{m} \mathrm{m}^{-1}$, only the seeds of S. grandiflora had germination of nearly $50 \%$ followed by $S$. siamea at about $20 \%$, while the other species had germination lower than $20 \%$. At the highest salt concentration $\left(40 \mathrm{dS} \mathrm{m}^{-1}\right)$, no seeds had a germinated percentage of more than $2 \%$ (Table 4 and Figure 3).

Table 3. Seed characteristics and storage behavior (seed size, shape, color, weight, and moisture content) in terms of the mean and standard deviation $( \pm \mathrm{SD})$

\begin{tabular}{|c|c|c|c|c|c|c|c|}
\hline \multirow[b]{2}{*}{ Species } & \multicolumn{2}{|c|}{ Size (mm) [Range] } & \multirow[b]{2}{*}{ Shape } & \multirow[b]{2}{*}{ Color } & \multirow{2}{*}{$\begin{array}{c}\text { Weight } \\
\text { (g/100 } \\
\text { seeds) }\end{array}$} & \multirow[b]{2}{*}{ Moisture (\%) } & \multirow[b]{2}{*}{$\begin{array}{l}\text { Storage } \\
\text { behavior }\end{array}$} \\
\hline & Length & Width & & & & & \\
\hline Adenanthera pavonina & $\begin{array}{l}9.09 \pm 0.41 \mathrm{def}^{*} \\
{[7.98-9.97]}\end{array}$ & $\begin{array}{l}9.60 \pm 0.39 b \\
{[8.61-10.63]}\end{array}$ & Heart & Red & $7.28 \pm 0.16 c$ & $8.74 \pm 0.28 \mathrm{ef}$ & Orthodox \\
\hline Albizia saman & $\begin{array}{l}9.25 \pm 0.67 d \\
{[7.78-10.71]}\end{array}$ & $\begin{array}{l}6.24 \pm 0.60 \mathrm{~g} \\
{[4.94-7.63]}\end{array}$ & Elliptic & Brown & $4.23 \pm 0.27 f$ & $9.67 \pm 0.55 \mathrm{de}$ & Orthodox \\
\hline Albizia procera & $\begin{array}{l}6.19 \pm 0.341 \\
{[5.20-6.89]}\end{array}$ & $\begin{array}{l}4.77 \pm 0.23 \mathrm{i} \\
{[4.19-5.55]}\end{array}$ & Elliptic & Yellow-Brown & $0.96 \pm 0.04 \mathrm{ij}$ & $12.21 \pm 0.59 b$ & Orthodox \\
\hline Cassia bakeriana & $\begin{array}{l}8.83 \pm 0.59 \mathrm{~g} \\
{[7.45-10.39]}\end{array}$ & $\begin{array}{l}6.72 \pm 0.42 \mathrm{e} \\
{[5.67-7.78]}\end{array}$ & Elliptic & Yellow-Brown & $5.38 \pm 0.20 \mathrm{~d}$ & $11.71 \pm 0.34 b c$ & Orthodox \\
\hline Cassia fistula & $\begin{array}{l}8.98 \pm 0.41 \mathrm{fg} \\
{[7.68-9.88]}\end{array}$ & $\begin{array}{l}6.88 \pm 0.31 \mathrm{~d} \\
{[6.04-7.77]}\end{array}$ & Elliptic & Yellow-Brown & $3.58 \pm 0.12 \mathrm{~g}$ & $12.65 \pm 0.11 b$ & Orthodox \\
\hline Cassia javanica & $\begin{array}{l}9.01 \pm 0.64 \mathrm{efg} \\
{[7.45-10.55]}\end{array}$ & $\begin{array}{l}6.89 \pm 0.54 \mathrm{~d} \\
{[5.67-8.42]}\end{array}$ & Elliptic & Yellow-Brown & $4.47 \pm 0.15 f$ & $7.43 \pm 0.27 \mathrm{~g}$ & Orthodox \\
\hline Dalbergia cochinchinensis & $\begin{array}{l}6.95 \pm 0.60 \mathrm{j} \\
{[3.68-8.22]}\end{array}$ & $\begin{array}{l}3.89 \pm 0.381 \\
{[2.98-4.89]}\end{array}$ & Kidney & Yellow-Brown & $0.70 \pm 0.03 \mathrm{j}$ & $11.98 \pm 0.51 b$ & Orthodox \\
\hline Dalbergia oliveri & $\begin{array}{l}11.53 \pm 0.94 c \\
{[9.37-13.87]}\end{array}$ & $\begin{array}{l}7.29 \pm 0.65 c \\
{[6.02-8.99]}\end{array}$ & Kidney & Yellow-Brown & $4.94 \pm 0.19 \mathrm{e}$ & $16.01 \pm 0.21 \mathrm{a}$ & Orthodox \\
\hline Delonix regia & $\begin{array}{l}18.46 \pm 1.05 b \\
{[15.45-21.06]}\end{array}$ & $\begin{array}{l}6.57 \pm 0.40 \mathrm{f} \\
{[5.61-7.50]}\end{array}$ & Oblong & Brown-Black & $11.79 \pm 0.39 b$ & $10.48 \pm 0.42 \mathrm{~cd}$ & Orthodox \\
\hline Millettia leucantha & $\begin{array}{l}19.47 \pm 1.83 \mathrm{a} \\
{[15.90-23.82]}\end{array}$ & $\begin{array}{l}11.42 \pm 0.87 \mathrm{a} \\
{[9.54-14.67]}\end{array}$ & Kidney & Brown & $14.65 \pm 1.02 \mathrm{a}$ & $9.54 \pm 3.14 \mathrm{de}$ & Orthodox \\
\hline Peltophorum dasyrrhachis & $\begin{array}{l}9.19 \pm 0.57 \mathrm{de} \\
{[7.84-10.54]}\end{array}$ & $\begin{array}{l}3.56 \pm 0.28 \mathrm{~m} \\
{[2.84-4.11]}\end{array}$ & Oblong & Yellow-Brown & $1.01 \pm 0.04 \mathrm{ij}$ & $8.27 \pm 0.25 f g$ & Orthodox \\
\hline Peltophorum pterocarpum & $\begin{array}{l}11.62 \pm 0.52 \mathrm{c} \\
{[10.09-13.06]}\end{array}$ & $\begin{array}{l}4.55 \pm 0.17 \mathrm{j} \\
{[4.11-5.03]}\end{array}$ & Oblong & Yellow-Brown & $1.65 \pm 0.06 \mathrm{~h}$ & $7.07 \pm 0.27 \mathrm{~g}$ & Orthodox \\
\hline Pterocarpus macrocarpus & $\begin{array}{l}8.33 \pm 0.71 \mathrm{~h} \\
{[6.49-10.45]}\end{array}$ & $\begin{array}{l}4.09 \pm 0.46 \mathrm{k} \\
{[3.16-5.01]}\end{array}$ & Kidney & Yellow-Brown & $1.34 \pm 0.08 \mathrm{hi}$ & $9.86 \pm 0.34 \mathrm{de}$ & Orthodox \\
\hline Senna siamea & $\begin{array}{l}7.21 \pm 0.53 \mathrm{i} \\
{[5.72-8.58]}\end{array}$ & $\begin{array}{l}5.88 \pm 0.39 h \\
{[4.81-6.86]}\end{array}$ & Elliptic & Brown & $0.64 \pm 0.05 \mathrm{j}$ & $10.49 \pm 0.93 \mathrm{~cd}$ & Orthodox \\
\hline Senna surattensis & $\begin{array}{l}6.66 \pm 0.37 \mathrm{k} \\
{[5.82-7.44]}\end{array}$ & $\begin{array}{l}3.34 \pm 0.34 \mathrm{n} \\
{[2.47-4.06]}\end{array}$ & Oblong & Brown & $0.70 \pm 0.02 \mathrm{j}$ & $10.22 \pm 0.45 \mathrm{~d}$ & Orthodox \\
\hline Sesbania grandiflora & $\begin{array}{l}5.75 \pm 0.34 \mathrm{~m} \\
{[4.65-6.59]}\end{array}$ & $\begin{array}{l}3.68 \pm 0.32 \mathrm{~m} \\
{[2.97-5.79]}\end{array}$ & Kidney & Yellow-Brown & $0.93 \pm 0.03 \mathrm{ij}$ & $12.72 \pm 0.20 b$ & Orthodox \\
\hline
\end{tabular}

Note: ${ }^{*}$ Mean values in each column followed by different letters indicate a significant difference at a probability level of 0.05 . 

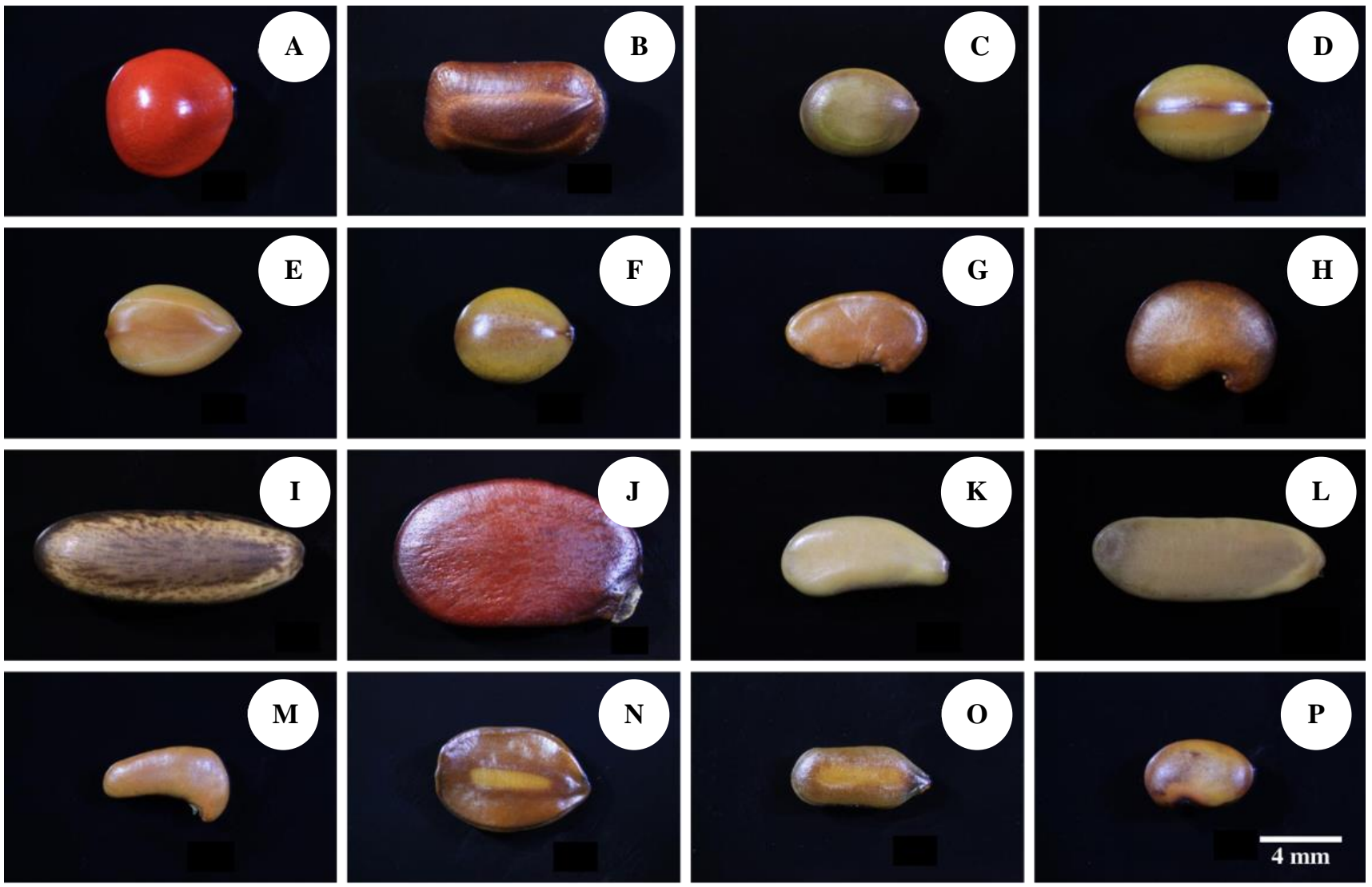

Figure 2. Images of (A) Adenanthera pavonina, (B) Albizia saman, (C) Albizia procera, (D) Cassia bakeriana, (E) Cassia fistula, (F) Cassia javanica, (G) Dalbergia cochinchinensis, (H) Dalbergia oliveri, (I) Delonix regia, (J) Millettia leucantha, (K) Peltophorum dasyrrhachis, (L) Peltophorum pterocarpum, (M) Pterocarpus macrocarpus, (N) Senna siamea, (O) Senna surattensis, and (P) Sesbania grandiflora

Mean germination time (MGT) describes the timespread of germination in unit of days. A low value of MGT indicates that the germination is faster when compared to a high value of MGT. Under the control, most seeds germinated rapidly between 2-4 days such as $S$. surattensis, $P$. macrocarpus, $S$. grandiflora, A. procera, $P$. pterocarpum, $S$. siamea, $D$. oliveri, $C$. javanica, $D$. cochinchinensis, and M. leucantha. The longest time taken to germinate was by $C$. fistula seeds at 18.58 days (Table 4). This indicates that its seeds may take more than 19 days for establishing as seedlings after sowing. Therefore, the preparation of $C$. fistula seedlings may take up to a month compared to other species which would take between 1-2 weeks only, under normal (no salinity) conditions.

Germination synchrony ( $Z$ ) describes the uniformity of germination. The highest value of $\mathrm{Z}$ is 1 and is reached when all the seeds germinate on the same day. Salinity can reduce the $\mathrm{Z}$-value due to a reduced ability of seeds to imbibe water under a saline solution. Under no salinity, the seeds of $S$. surattensis, $S$. grandiflora, $P$. macrocarpus, and $A$. procera had a high uniformity with a Z-value approximately between 0.6-0.7. Interestingly, the seeds of
$P$. dasyrrhachis under a salinity concentration of $24 \mathrm{dS} \mathrm{m}^{-1}$ had a Z-value $=1$. In this case, there were only 3 seeds of $P$. dasyrrhachis that could germinate in 1 day throughout the whole experiment of 30 days, resulting in germination synchrony of 1 , while the total germination was very low (Table 4).

In this study, the salinity level that reduced the germination by $50 \%\left(\mathrm{EC}_{50}\right)$ was used to compare the seed salt tolerance of the 16 species (Table 5). Four tolerance categories were constructed based on the value of $\mathrm{EC}_{50} . S$. grandiflora, $S$. siamea, and $D$. cochinchinensis were classified as highly salt-tolerant, indicated by $\mathrm{EC}_{50}$ values $\geq$ $30 \mathrm{dS} \mathrm{m}^{-1}$. In other words, salinity would have to be as high as $30 \mathrm{dS} \mathrm{m}$ m $^{-1}$ to reduce the germination of these seeds to $50 \%$. The high salt-tolerant group had $\mathrm{EC}_{50}$ values between $18-26 \mathrm{dS} \mathrm{m}^{-1}$ and included $A$. procera, $P$. macrocarpus, $S$. surattensis, A. saman, P. pterocarpum, C. fistula, and $P$. dasyrrhachis. The medium salt-tolerant group had $\mathrm{EC}_{50}$ values between 9-16 dS m $\mathrm{m}^{-1}$ and included $C$. bakeriana, $D$. regia, and $M$. leucantha. The last group comprised of the low salt-tolerant species with $\mathrm{EC}_{50}$ value $<8 \mathrm{dS} \mathrm{m}^{-1}$ and included $C$. javanica, D. oliveri, and A. pavonina. 
Table 4. Germination percentage (GP), mean germination time (MGT), germination speed (GS), and germination synchrony (Z)

\begin{tabular}{|c|c|c|c|c|c|}
\hline Species & $\mathrm{NaCl}\left(\mathrm{dS} \mathrm{m} \mathbf{m}^{-1}\right)$ & GP $(\%)$ & MGT (day) & GS $\left(\right.$ seed day $\left.{ }^{-1}\right)$ & $\mathrm{Z}$ (unit less) \\
\hline \multirow[t]{7}{*}{ Adenanthera pavonina } & 0 & $18.00 \pm 6.93 \mathrm{a}$ & $6.33 \pm 0.88 \mathrm{~b}$ & $0.73 \pm 0.22 \mathrm{a}$ & $0.41 \pm 0.40 \mathrm{a}$ \\
\hline & 8 & $0.00 \pm 0.00 \mathrm{~b}$ & $30.00 \pm 0.00 \mathrm{a}$ & $0.00 \pm 0.00 \mathrm{~b}$ & $0.00 \pm 0.00 \mathrm{~b}$ \\
\hline & 16 & $0.00 \pm 0.00 \mathrm{~b}$ & $30.00 \pm 0.00 \mathrm{a}$ & $0.00 \pm 0.00 \mathrm{~b}$ & $0.00 \pm 0.00 \mathrm{~b}$ \\
\hline & 24 & $0.00 \pm 0.00 \mathrm{~b}$ & $30.00 \pm 0.00 \mathrm{a}$ & $0.00 \pm 0.00 \mathrm{~b}$ & $0.00 \pm 0.00 \mathrm{~b}$ \\
\hline & 32 & $0.00 \pm 0.00 \mathrm{~b}$ & $30.00 \pm 0.00 \mathrm{a}$ & $0.00 \pm 0.00 \mathrm{~b}$ & $0.00 \pm 0.00 \mathrm{~b}$ \\
\hline & 40 & $0.00 \pm 0.00 \mathrm{~b}$ & $30.00 \pm 0.00 \mathrm{a}$ & $0.00 \pm 0.00 \mathrm{~b}$ & $0.00 \pm 0.00 \mathrm{~b}$ \\
\hline & $P$-value & $<0.001$ & $<0.001$ & $<0.001$ & 0.0107 \\
\hline \multirow[t]{7}{*}{ Albizia saman } & 0 & $33.00 \pm 39.92 \mathrm{ab}$ & $10.15 \pm 5.03 \mathrm{c}$ & $1.26 \pm 1.06 \mathrm{ab}$ & $0.05 \pm 0.08 \mathrm{a}$ \\
\hline & 8 & $48.00 \pm 32.33 \mathrm{a}$ & $17.24 \pm 5.09 \mathrm{bc}$ & $1.68 \pm 1.02 \mathrm{a}$ & $0.09 \pm 0.07 \mathrm{a}$ \\
\hline & 16 & $17.00 \pm 8.25 \mathrm{abc}$ & $10.51 \pm 4.02 \mathrm{c}$ & $0.57 \pm 0.28 \mathrm{bc}$ & $0.05 \pm 0.08 \mathrm{a}$ \\
\hline & 24 & $17.00 \pm 10.52 \mathrm{abc}$ & $12.35 \pm 5.13 \mathrm{c}$ & $0.52 \pm 0.23 \mathrm{bc}$ & $0.10 \pm 0.16 \mathrm{a}$ \\
\hline & 32 & $6.00 \pm 5.16 \mathrm{bc}$ & $23.63 \pm 7.43 \mathrm{ab}$ & $0.10 \pm 0.10 \mathrm{c}$ & $0.00 \pm 0.00 \mathrm{a}$ \\
\hline & 40 & $0.00 \pm 0.00 \mathrm{c}$ & $30.00 \pm 0.00 \mathrm{a}$ & $0.00 \pm 0.00 \mathrm{c}$ & $0.00 \pm 0.00 \mathrm{a}$ \\
\hline & $P$-value & 0.058 & 0.0181 & 0.0078 & 0.433 \\
\hline \multirow{7}{*}{ Albizia procera } & 0 & $71.00 \pm 13.61 \mathrm{a}$ & $2.66 \pm 0.38 \mathrm{~b}$ & $7.79 \pm 1.93 \mathrm{a}$ & $0.59 \pm 0.17 \mathrm{a}$ \\
\hline & 8 & $56.00 \pm 7.30 \mathrm{ab}$ & $2.42 \pm 0.25 \mathrm{~b}$ & $6.41 \pm 1.14 \mathrm{ab}$ & $0.67 \pm 0.10 \mathrm{a}$ \\
\hline & 16 & $43.00 \pm 18.00 \mathrm{~b}$ & $2.65 \pm 0.42 \mathrm{~b}$ & $4.67 \pm 2.03 \mathrm{~b}$ & $0.52 \pm 0.15 \mathrm{a}$ \\
\hline & 24 & $55.00 \pm 13.61 \mathrm{ab}$ & $2.96 \pm 0.70 \mathrm{~b}$ & $5.52 \pm 1.53 \mathrm{~b}$ & $0.39 \pm 0.14 \mathrm{a}$ \\
\hline & 32 & $8.00 \pm 5.66 \mathrm{c}$ & $6.50 \pm 1.73 \mathrm{~b}$ & $0.37 \pm 0.32 \mathrm{c}$ & $0.50 \pm 0.58 \mathrm{a}$ \\
\hline & 40 & $1.00 \pm 2.00 \mathrm{c}$ & $23.75 \pm 12.50 \mathrm{a}$ & $0.05 \pm 0.10 \mathrm{c}$ & $0.00 \pm 0.00 \mathrm{~b}$ \\
\hline & $P$-value & $<0.001$ & $<0.001$ & $<0.001$ & 0.0275 \\
\hline \multirow[t]{7}{*}{ Cassia bakeriana } & 0 & $11.00 \pm 6.00 \mathrm{a}$ & $4.90 \pm 2.52 \mathrm{~b}$ & $0.86 \pm 0.76 \mathrm{a}$ & $0.10 \pm 0.20 \mathrm{a}$ \\
\hline & 8 & $6.00 \pm 5.16 \mathrm{abc}$ & $13.29 \pm 11.35 b$ & $0.36 \pm 0.42 \mathrm{ab}$ & $0.00 \pm 0.00 \mathrm{a}$ \\
\hline & 16 & $7.00 \pm 6.83 \mathrm{ab}$ & $15.25 \pm 9.94 \mathrm{~b}$ & $0.24 \pm 0.30 \mathrm{~b}$ & $0.25 \pm 0.50 \mathrm{a}$ \\
\hline & 24 & $4.00 \pm 3.27 \mathrm{~b} \mathrm{c}$ & $13.00 \pm 11.58 \mathrm{~b}$ & $0.15 \pm 0.11 \mathrm{~b}$ & $0.00 \pm 0.00 \mathrm{a}$ \\
\hline & 32 & $0.00 \pm 0.00 \mathrm{c}$ & $30.00 \pm 0.00 \mathrm{a}$ & $0.00 \pm 0.00 \mathrm{~b}$ & $0.00 \pm 0.00 \mathrm{a}$ \\
\hline & 40 & $0.00 \pm 0.00 \mathrm{c}$ & $30.00 \pm 0.00 \mathrm{a}$ & $0.00 \pm 0.00 \mathrm{~b}$ & $0.00 \pm 0.00 \mathrm{a}$ \\
\hline & $P$-value & 0.019 & 0.0228 & 0.0436 & 0.525 \\
\hline \multirow[t]{7}{*}{ Cassia fistula } & 0 & $13.00 \pm 3.83 \mathrm{a}$ & $18.58 \pm 2.51 \mathrm{~b}$ & $0.34 \pm 0.28 \mathrm{a}$ & $0.04 \pm 0.08 \mathrm{a}$ \\
\hline & 8 & $13.00 \pm 6.83 \mathrm{a}$ & $20.22 \pm 5.50 \mathrm{~b}$ & $0.25 \pm 0.22 \mathrm{ab}$ & $0.15 \pm 0.24 \mathrm{a}$ \\
\hline & 16 & $9.00 \pm 2.00 \mathrm{ab}$ & $21.58 \pm 6.03 \mathrm{~b}$ & $0.23 \pm 0.22 \mathrm{ab}$ & $0.00 \pm 0.00 \mathrm{a}$ \\
\hline & 24 & $4.00 \pm 3.27 \mathrm{bc}$ & $21.13 \pm 10.93 \mathrm{~b}$ & $0.07 \pm 0.06 \mathrm{~b}$ & $0.00 \pm 0.00 \mathrm{a}$ \\
\hline & 32 & $0.00 \pm 0.00 \mathrm{c}$ & $30.00 \pm 0.00 \mathrm{a}$ & $0.00 \pm 0.00 \mathrm{~b}$ & $0.00 \pm 0.00 \mathrm{a}$ \\
\hline & 40 & $0.00 \pm 0.00 \mathrm{c}$ & $30.00 \pm 0.00 \mathrm{a}$ & $0.00 \pm 0.00 \mathrm{~b}$ & $0.00 \pm 0.00 \mathrm{a}$ \\
\hline & $P$-value & $<0.001$ & $<0.001$ & 0.0026 & 0.283 \\
\hline \multirow[t]{7}{*}{ Cassia javanica } & 0 & $9.00 \pm 6.00 \mathrm{a}$ & $9.59 \pm 13.63 \mathrm{~b}$ & $0.95 \pm 0.66 \mathrm{a}$ & $0.42 \pm 0.42 \mathrm{a}$ \\
\hline & 8 & $4.00 \pm 3.27 \mathrm{~b}$ & $10.00 \pm 13.34 \mathrm{~b}$ & $0.33 \pm 0.31 \mathrm{~b}$ & $0.00 \pm 0.00 \mathrm{~b}$ \\
\hline & 16 & $4.00 \pm 3.27 \mathrm{~b}$ & $12.00 \pm 12.08 \mathrm{~b}$ & $0.19 \pm 0.15 \mathrm{~b}$ & $0.00 \pm 0.00 \mathrm{~b}$ \\
\hline & 24 & $3.00 \pm 2.00 \mathrm{~b}$ & $14.25 \pm 10.59 \mathrm{~b}$ & $0.09 \pm 0.06 \mathrm{~b}$ & $0.00 \pm 0.00 \mathrm{~b}$ \\
\hline & 32 & $0.00 \pm 0.00 \mathrm{~b}$ & $30.00 \pm 0.00 \mathrm{a}$ & $0.00 \pm 0.00 \mathrm{~b}$ & $0.00 \pm 0.00 \mathrm{~b}$ \\
\hline & 40 & $0.00 \pm 0.00 \mathrm{~b}$ & $30.00 \pm 0.00 \mathrm{a}$ & $0.00 \pm 0.00 \mathrm{~b}$ & $0.00 \pm 0.00 \mathrm{~b}$ \\
\hline & $P$-value & 0.009 & 0.0095 & 0.0646 & 0.0142 \\
\hline \multirow{7}{*}{ Dalbergia cochinchinensis } & 0 & $31.00 \pm 13.22 \mathrm{ab}$ & $2.26 \pm 0.24 \mathrm{e}$ & $6.07 \pm 1.59 \mathrm{a}$ & $0.38 \pm 0.08 \mathrm{a}$ \\
\hline & 8 & $43.00 \pm 12.81 \mathrm{a}$ & $3.01 \pm 0.41 \mathrm{~d}$ & $3.94 \pm 0.42 \mathrm{~b}$ & $0.44 \pm 0.30 \mathrm{a}$ \\
\hline & 16 & $37.00 \pm 18.29 \mathrm{a}$ & $4.14 \pm 0.74 \mathrm{c}$ & $2.70 \pm 0.68 \mathrm{c}$ & $0.29 \pm 0.07 \mathrm{ab}$ \\
\hline & 24 & $28.00 \pm 18.18 \mathrm{ab}$ & $5.94 \pm 0.63 \mathrm{~b}$ & $0.88 \pm 0.23 \mathrm{~d}$ & $0.13 \pm 0.14 b c$ \\
\hline & 32 & $10.00 \pm 20.00 \mathrm{bc}$ & $30.00 \pm 2.00 \mathrm{a}$ & $0.06 \pm 0.13 \mathrm{~d}$ & $0.00 \pm 0.00 \mathrm{c}$ \\
\hline & 40 & $0.00 \pm 0.00 \mathrm{c}$ & $30.00 \pm 0.00 \mathrm{a}$ & $0.00 \pm 0.00 \mathrm{~d}$ & $0.00 \pm 0.00 \mathrm{c}$ \\
\hline & $P$-value & 0.007 & $<0.001$ & $<0.001$ & $<0.001$ \\
\hline \multirow[t]{7}{*}{ Dalbergia oliveri } & 0 & $2.00 \pm 4.00 \mathrm{a}$ & $23.75 \pm 12.50 \mathrm{a}$ & $0.12 \pm 0.24 \mathrm{a}$ & $0.00 \pm 0.00 \mathrm{a}$ \\
\hline & 8 & $1.00 \pm 2.00 \mathrm{a}$ & $25.50 \pm 9.00 \mathrm{a}$ & $0.02 \pm 0.04 \mathrm{a}$ & $0.00 \pm 0.00 \mathrm{a}$ \\
\hline & 16 & $0.00 \pm 0.00 \mathrm{a}$ & $30.00 \pm 0.00 \mathrm{a}$ & $0.00 \pm 0.00 \mathrm{a}$ & $0.00 \pm 0.00 \mathrm{a}$ \\
\hline & 24 & $0.00 \pm 0.00 \mathrm{a}$ & $30.00 \pm 0.00 \mathrm{a}$ & $0.00 \pm 0.00 \mathrm{a}$ & $0.00 \pm 0.00 \mathrm{a}$ \\
\hline & 32 & $0.00 \pm 0.00 \mathrm{a}$ & $30.00 \pm 0.00 \mathrm{a}$ & $0.00 \pm 0.00 \mathrm{a}$ & $0.00 \pm 0.00 \mathrm{a}$ \\
\hline & 40 & $0.00 \pm 0.00 \mathrm{a}$ & $30.00 \pm 0.00 \mathrm{a}$ & $0.00 \pm 0.00 \mathrm{a}$ & $0.00 \pm 0.00 \mathrm{a}$ \\
\hline & $P$-value & 0.539 & 0.528 & 0.482 & - \\
\hline \multirow[t]{7}{*}{ Delonix regia } & 0 & $46.00 \pm 14.05 \mathrm{a}$ & $4.15 \pm 0.88 \mathrm{~b}$ & $3.41 \pm 1.39 \mathrm{a}$ & $0.20 \pm 0.07 \mathrm{a}$ \\
\hline & 8 & $42.00 \pm 13.66 \mathrm{a}$ & $5.50 \pm 1.30 \mathrm{~b}$ & $2.24 \pm 0.69 \mathrm{~b}$ & $0.17 \pm 0.07 \mathrm{a}$ \\
\hline & 16 & $14.00 \pm 9.52 \mathrm{~b}$ & $11.85 \pm 12.13 \mathrm{~b}$ & $0.64 \pm 0.44 \mathrm{c}$ & $0.17 \pm 0.12 \mathrm{a}$ \\
\hline & 24 & $1.00 \pm 2.00 \mathrm{bc}$ & $25.00 \pm 10.00 \mathrm{a}$ & $0.03 \pm 0.05 \mathrm{c}$ & $0.00 \pm 0.00 \mathrm{~b}$ \\
\hline & 32 & $0.00 \pm 0.00 \mathrm{c}$ & $30.00 \pm 0.00 \mathrm{a}$ & $0.00 \pm 0.00 \mathrm{c}$ & $0.00 \pm 0.00 \mathrm{~b}$ \\
\hline & 40 & $0.00 \pm 0.00 \mathrm{c}$ & $30.00 \pm 0.00 \mathrm{a}$ & $0.00 \pm 0.00 \mathrm{c}$ & $0.00 \pm 0.00 \mathrm{~b}$ \\
\hline & $P$-value & $<0.001$ & 0.0202 & $<0.001$ & $<0.001$ \\
\hline
\end{tabular}




\begin{tabular}{|c|c|c|c|c|c|}
\hline \multirow[t]{7}{*}{ Millettia leucantha } & 0 & $35.00 \pm 18.30 \mathrm{a}$ & $2.41 \pm 0.67 \mathrm{c}$ & $4.05 \pm 1.65 \mathrm{a}$ & $0.32 \pm 0.10 \mathrm{a}$ \\
\hline & 8 & $20.00 \pm 13.86 \mathrm{~b}$ & $2.81 \pm 0.63 \mathrm{c}$ & $2.05 \pm 1.39 \mathrm{~b}$ & $0.43 \pm 0.41 \mathrm{a}$ \\
\hline & 16 & $8.00 \pm 5.66 \mathrm{bc}$ & $4.81 \pm 2.54 \mathrm{c}$ & $0.93 \pm 1.21 \mathrm{bc}$ & $0.04 \pm 0.08 \mathrm{~b}$ \\
\hline & 24 & $2.00 \pm 2.31 \mathrm{c}$ & $17.25 \pm 14.73 \mathrm{bc}$ & $0.11 \pm 0.13 \mathrm{c}$ & $0.00 \pm 0.00 \mathrm{~b}$ \\
\hline & 32 & $0.00 \pm 0.00 \mathrm{c}$ & $30.00 \pm 0.00 \mathrm{a}$ & $0.00 \pm 0.00 \mathrm{c}$ & $0.00 \pm 0.00 \mathrm{~b}$ \\
\hline & 40 & $0.00 \pm 0.00 \mathrm{c}$ & $30.00 \pm 0.00 \mathrm{a}$ & $0.00 \pm 0.00 \mathrm{c}$ & $0.00 \pm 0.00 \mathrm{~b}$ \\
\hline & $P$-value & $<0.001$ & 0.0026 & $<0.001$ & 0.0066 \\
\hline \multirow{7}{*}{ Peltophorum dasyrrhachis } & 0 & $91.00 \pm 3.82 \mathrm{a}$ & $2.92 \pm 0.11 \mathrm{e}$ & $8.18 \pm 0.27 \mathrm{a}$ & $0.46 \pm 0.06 \mathrm{~b}$ \\
\hline & 8 & $95.00 \pm 3.83 \mathrm{a}$ & $5.06 \pm 0.25 \mathrm{~d}$ & $4.95 \pm 0.14 \mathrm{~b}$ & $0.25 \pm 0.06 \mathrm{c}$ \\
\hline & 16 & $78.00 \pm 18.04 \mathrm{~b}$ & $9.39 \pm 2.35 \mathrm{c}$ & $2.17 \pm 0.42 \mathrm{c}$ & $0.21 \pm 0.11 \mathrm{c}$ \\
\hline & 24 & $12.00 \pm 5.66 \mathrm{c}$ & $11.50 \pm 1.00 \mathrm{~b}$ & $0.26 \pm 0.11 \mathrm{~d}$ & $1.00 \pm 0.00 \mathrm{a}$ \\
\hline & 32 & $0.00 \pm 0.00 \mathrm{~d}$ & $30.00 \pm 0.00 \mathrm{a}$ & $0.00 \pm 0.00 \mathrm{~d}$ & $0.00 \pm 0.00 \mathrm{~d}$ \\
\hline & 40 & $0.00 \pm 0.00 \mathrm{~d}$ & $30.00 \pm 0.00 \mathrm{a}$ & $0.00 \pm 0.00 \mathrm{~d}$ & $0.00 \pm 0.00 \mathrm{~d}$ \\
\hline & $P$-value & $<0.001$ & $<0.001$ & $<0.001$ & $<0.001$ \\
\hline \multirow{7}{*}{ Peltophorum pterocarpum } & 0 & $74.00 \pm 19.18 \mathrm{a}$ & $2.34 \pm 0.33 \mathrm{c}$ & $9.77 \pm 1.92 \mathrm{a}$ & $0.64 \pm 0.21 \mathrm{a}$ \\
\hline & 8 & $89.00 \pm 8.87 \mathrm{a}$ & $2.95 \pm 0.28 \mathrm{c}$ & $8.32 \pm 1.17 \mathrm{a}$ & $0.35 \pm 0.07 \mathrm{ab}$ \\
\hline & 16 & $74.00 \pm 10.58 \mathrm{a}$ & $4.87 \pm 0.53 \mathrm{c}$ & $3.98 \pm 0.61 \mathrm{~b}$ & $0.30 \pm 0.15 \mathrm{ab}$ \\
\hline & 24 & $29.00 \pm 21.51 \mathrm{~b}$ & $12.94 \pm 11.45 \mathrm{~b}$ & $1.07 \pm 0.90 \mathrm{c}$ & $0.33 \pm 0.45 \mathrm{bc}$ \\
\hline & 32 & $0.00 \pm 0.00 \mathrm{c}$ & $30.00 \pm 0.00 \mathrm{a}$ & $0.00 \pm 0.00 \mathrm{c}$ & $0.00 \pm 0.00 \mathrm{c}$ \\
\hline & 40 & $0.00 \pm 0.00 \mathrm{c}$ & $30.00 \pm 0.00 \mathrm{a}$ & $0.00 \pm 0.00 \mathrm{c}$ & $0.00 \pm 0.00 \mathrm{c}$ \\
\hline & $P$-value & $<0.001$ & $<0.001$ & $<0.001$ & 0.0043 \\
\hline \multirow[t]{7}{*}{ Pterocarpus macrocarpus } & 0 & $64.00 \pm 9.80 \mathrm{a}$ & $5.94 \pm 0.85 \mathrm{~b}$ & $3.74 \pm 0.69 \mathrm{a}$ & $0.10 \pm 0.03 \mathrm{a}$ \\
\hline & 8 & $55.00 \pm 5.03 \mathrm{a}$ & $7.80 \pm 1.20 \mathrm{~b}$ & $2.64 \pm 0.27 \mathrm{~b}$ & $0.10 \pm 0.03 \mathrm{a}$ \\
\hline & 16 & $31.00 \pm 6.83 \mathrm{~b}$ & $9.62 \pm 1.58 \mathrm{~b}$ & $1.10 \pm 0.13 \mathrm{c}$ & $0.03 \pm 0.04 \mathrm{a}$ \\
\hline & 24 & $30.00 \pm 12.44 \mathrm{~b}$ & $11.05 \pm 1.55 \mathrm{~b}$ & $0.87 \pm 0.56 \mathrm{c}$ & $0.04 \pm 0.05 \mathrm{a}$ \\
\hline & 32 & $9.00 \pm 3.83 \mathrm{c}$ & $13.04 \pm 2.54 \mathrm{~b}$ & $0.19 \pm 0.12 \mathrm{~d}$ & $0.08 \pm 0.17 \mathrm{a}$ \\
\hline & 40 & $1.00 \pm 2.00 \mathrm{c}$ & $24.25 \pm 11.50 \mathrm{a}$ & $0.04 \pm 0.07 \mathrm{~d}$ & $0.00 \pm 0.00 \mathrm{a}$ \\
\hline & $P$-value & $<0.001$ & $<0.001$ & $<0.001$ & 0.422 \\
\hline \multirow[t]{7}{*}{ Senna siamea } & 0 & $51.00 \pm 11.94 \mathrm{ab}$ & $3.65 \pm 0.31 \mathrm{c}$ & $5.19 \pm 1.42 \mathrm{a}$ & $0.48 \pm 0.16 \mathrm{a}$ \\
\hline & 8 & $59.00 \pm 12.81 \mathrm{a}$ & $5.48 \pm 1.56 \mathrm{c}$ & $5.42 \pm 1.38 \mathrm{a}$ & $0.35 \pm 0.11 \mathrm{a}$ \\
\hline & 16 & $37.00 \pm 16.12 \mathrm{bc}$ & $11.24 \pm 1.57 \mathrm{~b}$ & $1.54 \pm 0.77 \mathrm{bc}$ & $0.06 \pm 0.05 \mathrm{~b}$ \\
\hline & 24 & $67.00 \pm 8.87 \mathrm{a}$ & $11.30 \pm 2.45 \mathrm{~b}$ & $2.05 \pm 0.55 \mathrm{~b}$ & $0.12 \pm 0.03 \mathrm{~b}$ \\
\hline & 32 & $20.00 \pm 14.61 \mathrm{c}$ & $13.09 \pm 1.69 \mathrm{~b}$ & $0.39 \pm 0.29 \mathrm{~cd}$ & $0.14 \pm 0.15 b$ \\
\hline & 40 & $0.00 \pm 0.00 \mathrm{~d}$ & $30.00 \pm 0.00 \mathrm{a}$ & $0.00 \pm 0.00 \mathrm{~d}$ & $0.00 \pm 0.00 \mathrm{~b}$ \\
\hline & $P$-value & $<0.001$ & $<0.001$ & $<0.001$ & $<0.001$ \\
\hline \multirow[t]{7}{*}{ Senna surattensis } & 0 & $75.00 \pm 6.83 \mathrm{a}$ & $2.18 \pm 0.14 \mathrm{c}$ & $8.79 \pm 0.44 \mathrm{a}$ & $0.72 \pm 0.16 \mathrm{a}$ \\
\hline & 8 & $72.00 \pm 3.27 \mathrm{a}$ & $2.48 \pm 0.27 \mathrm{c}$ & $7.92 \pm 0.50 \mathrm{~b}$ & $0.55 \pm 0.09 \mathrm{ab}$ \\
\hline & 16 & $66.00 \pm 5.16 \mathrm{a}$ & $3.41 \pm 0.32 \mathrm{c}$ & $5.28 \pm 0.32 \mathrm{c}$ & $0.54 \pm 0.17 \mathrm{ab}$ \\
\hline & 24 & $30.25 \pm 15.88 \mathrm{~b}$ & $10.64 \pm 3.91 \mathrm{~b}$ & $0.93 \pm 0.54 \mathrm{~d}$ & $0.06 \pm 0.06 \mathrm{c}$ \\
\hline & 32 & $3.00 \pm 6.00 \mathrm{c}$ & $24.75 \pm 10.50 \mathrm{a}$ & $0.08 \pm 0.17 \mathrm{e}$ & $0.25 \pm 0.50 \mathrm{bc}$ \\
\hline & 40 & $0.00 \pm 0.00 \mathrm{c}$ & $30.00 \pm 0.00 \mathrm{a}$ & $0.00 \pm 0.00 \mathrm{e}$ & $0.00 \pm 0.00 \mathrm{c}$ \\
\hline & $P$-value & $<0.001$ & $<0.001$ & $<0.001$ & 0.0012 \\
\hline \multirow[t]{7}{*}{ Sesbania grandiflora } & 0 & $83.00 \pm 7.57 \mathrm{a}$ & $2.43 \pm 0.39 \mathrm{~d}$ & $9.52 \pm 1.44 \mathrm{a}$ & $0.71 \pm 0.25 \mathrm{a}$ \\
\hline & 8 & $82.00 \pm 12.00 \mathrm{a}$ & $2.64 \pm 0.16 \mathrm{~d}$ & $8.40 \pm 1.55 \mathrm{a}$ & $0.44 \pm 0.05 \mathrm{~b}$ \\
\hline & 16 & $88.00 \pm 5.66 \mathrm{a}$ & $3.72 \pm 0.43 \mathrm{~cd}$ & $6.42 \pm 0.69 \mathrm{~b}$ & $0.45 \pm 0.11 \mathrm{~b}$ \\
\hline & 24 & $83.00 \pm 11.49 \mathrm{a}$ & $7.10 \pm 0.70 \mathrm{c}$ & $3.34 \pm 0.58 \mathrm{c}$ & $0.15 \pm 0.04 \mathrm{c}$ \\
\hline & 32 & $47.00 \pm 5.03 \mathrm{~b}$ & $14.36 \pm 1.23 \mathrm{~b}$ & $0.90 \pm 0.16 \mathrm{~d}$ & $0.12 \pm 0.07 \mathrm{c}$ \\
\hline & 40 & $1.00 \pm 2.00 \mathrm{c}$ & $27.00 \pm 6.00 \mathrm{a}$ & $0.02 \pm 0.03 \mathrm{~d}$ & $0.00 \pm 0.00 \mathrm{c}$ \\
\hline & $P$-value & $<0.001$ & 0.0021 & $<0.001$ & $<0.001$ \\
\hline
\end{tabular}

Note: Different letters in each column indicate the mean difference among salinity concentration in each species analyzed with ANOVA at a significant level of $P<0.05$

\section{Discussion}

Species selection is a key for successful restoration of salt-affected areas. Not only do the species need to have salt tolerance, but they also need to have some economic value such that they can be recommended to a farmer as a viable income source as well. In this context, Fabaceae species have the potential to solve these problems, not only because they can help in sustainably repairing the degraded environments, but also deliver economic benefits. These functions are largely supported by the physiological traits of the seeds of fabaceous species. Most seeds in the Fabaceae family have epigeal germination (Jayasuriya et al. 2010; Yahara et al. 2013) in which the cotyledon appears above the soil surface with the help of an elongated hypocotyl and serves to help synthesize light for photosynthetic process (Perez-Harguindeguy et al. 2013). This might be a partial reason for the fast establishment and growth of species in Fabaceae family. 
Seed moisture content (SMC) is the percentage of water held by a seed. Therefore, seed water content may be an important factor affecting the water absorption and the subsequent germination response (Long et al. 2010). It is not only a critical factor in determining seed storage behavior because seeds tend to deteriorate rapidly when they have a high SMC, but also affects the germination as it controls the rate of water imbibition during the initial stages of germination (Bewley and Black 2012; Baskin and Baskin 2014). Most of the orthodox seeds have a hard shell, resulting in only a slight change in seed moisture (Bewley and Black 2012). Most seeds of the dominant tropical tree species are classified as recalcitrant while most of the seeds from the fabaceous species are orthodox (Yahara et al. 2013). A recalcitrant seed has short life span due to a high SMC (20-50\%). Our result was similar to Jayasuriya et al. (2013), who studied seed germination of Fabaceae family in Sri Lanka and reported that seeds of 94 species (out of 100) had an orthodox behavior with an average SMC less than $16 \%$. In contrast to orthodox seeds, the remaining 6 species had recalcitrant seeds with SMC between 29.8-57.1\% (Jayasuriya et al. 2013).
Table 5. Electrical conductivity resulting in a reduction in germination by $50 \%$ (EC50) compared among the seeds of 16 fabaceous species

\begin{tabular}{lll}
\hline Species & $\begin{array}{c}\text { EC50 } \\
\left(\mathbf{d S} \mathbf{~ m}^{-1}\right)\end{array}$ & $\begin{array}{c}\text { Level of } \\
\text { tolerance }\end{array}$ \\
\hline Sesbania grandiflora & 33.56 & Highest \\
Senna siamea & 32.93 & Highest \\
Dalbergia cochinchinensis & 30.83 & Highest \\
Albizia procera & 25.74 & High \\
Peltophorum pterocarpum & 22.40 & High \\
Senna surattensis & 21.97 & High \\
Albizia saman & 21.67 & High \\
Pterocarpus macrocarpus & 18.97 & High \\
Cassia fistula & 18.73 & High \\
Peltophorum dasyrrhachis & 18.57 & High \\
Cassia bakeriana & 16.08 & Medium \\
Delonix regia & 13.47 & Medium \\
Millettia leucantha & 9.35 & Medium \\
Dalbergia oliveri & 6.85 & Low \\
Cassia javanica & 5.45 & Low \\
Adenanthera pavonina & 0.35 & Low \\
\hline Note: The level of torand
\end{tabular}

Note: The level of tolerance is based on the salinity indicated in a soil solution proposed by US Salinity Laboratory Staff (1954).
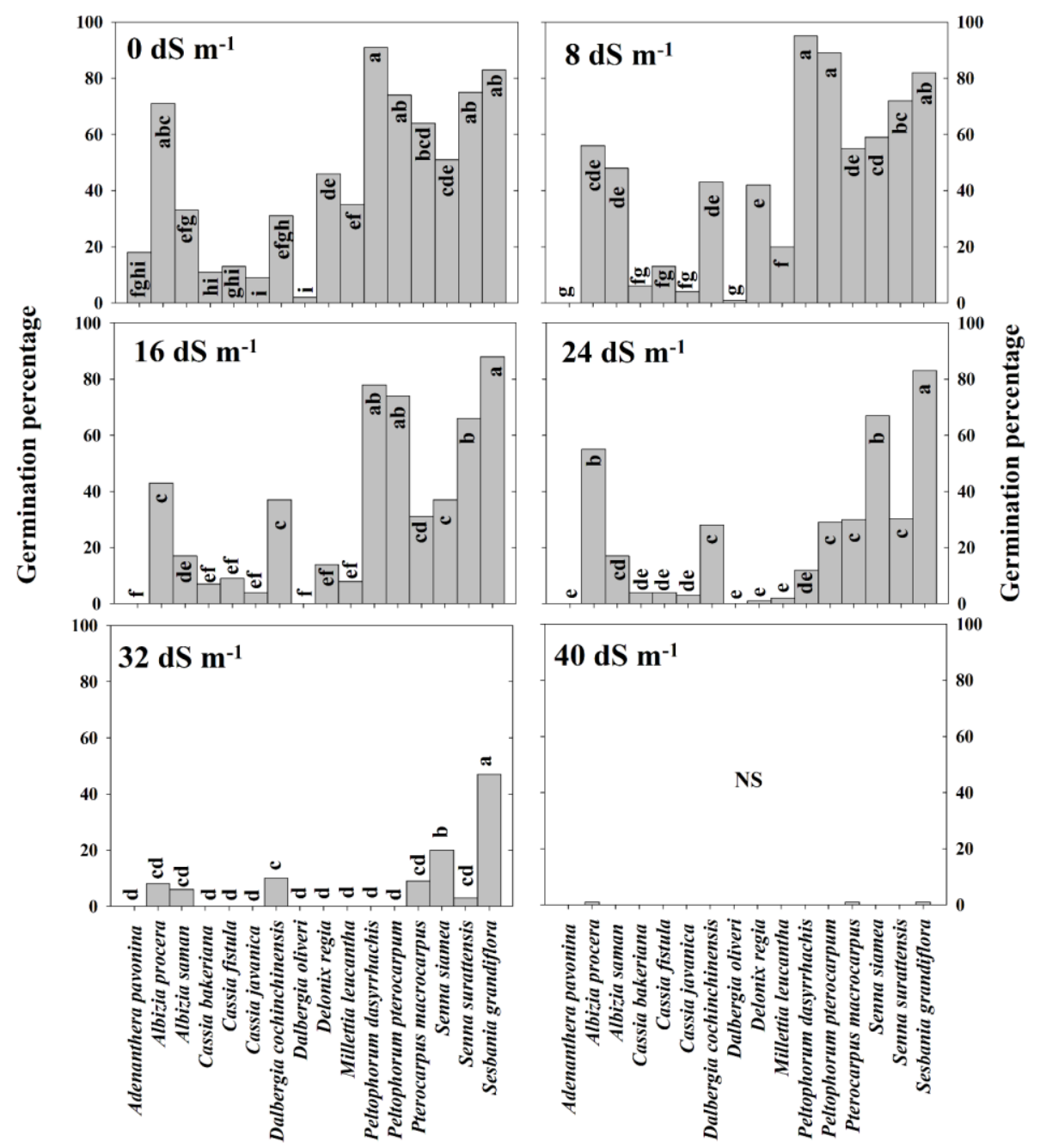

Figure 3. Total pool germination percentage of each species. The different letters above each bar indicate the mean difference among species at a significant level of 0.05 
Most of the seeds in our study germinated to more than $40 \%$ under zero salinity (control treatment). However, some species had a low seed germination, especially $D$. oliveri. Most of the fabaceous seeds are physically dormant (Jayasuriya et al. 2010; Jaganathan et al. 2017). The fabaceous seed is dormant because the impermeability of the testa, which acts as a barrier to seed's imbibition or physical dormancy (Gunes and Ali 2011). To break the dormancy, in this study, all seeds were soaked in hot water and then immersed overnight in water maintained at room temperature. Even after following this procedure, the germination percentage of $D$. oliveri was still low. This indicated that the seed preparation process might not be suitable for this species due to a very thick seed coat observed in the seeds of $D$. oliveri. According to Jayasuriya et al. (2013), seeds of Dalbergia lanceolaria should be primed with 100 or 500 ppm GA3 (Gibberellic acid) before germination test. In addition, seeds of 3 species in the genus Cassia had a low germination percentage as well. Typically, most Cassia seeds are physical dormant (Schmidt, 2007). Vozzo (2002) suggested that acid scarification could help break the seed dormancy in small seeds of tropical species such as Acacia, Cassia, Senna, Sesbania, and Tamarindus. Todaria and Negi (1992) studied how to break seed dormancy in Cassia species in India and indicated that seed germination improved after acid scarification using sulfuric acid $\left(\mathrm{H}_{2} \mathrm{SO}_{4}\right)$. Another study by Srivastava et al. (2012) modified the seed dormancy breaking method from Todaria and Negi (1992) by soaking the seeds in $98 \%$ concentrated sulfuric acid for 40 minutes before sowing. This increased the germination percentage of Cassia occidentalis seeds by $40 \%$.

All the seeds tested in this study germinated well under a salinity of $0-16 \mathrm{dS} \mathrm{m} \mathrm{m}^{-1}$. However, the yearly average salinity levels in the northeastern part of Thailand, at a soil depth of $0-0.1 \mathrm{~m}$, have been reported at $24 \mathrm{dS} \mathrm{m} \mathrm{m}^{-1}$ (Leksungnoen 2017). When the level of salinity was increased to this value, the germination reduced significantly compared to that at a salinity between $0-16 \mathrm{dS}$ $\mathrm{m}^{-1}$. However, $S$. grandiflora seeds had a high germination of $83 \%$ followed by $S$. siamea and A. Procera, which had a germination of more than $50 \%$, while $S$. surattensis, $P$. macrocarpus, $P$. pterocarpum, and D. cochinchinensis had a reasonable germination of $30,30,30,29$, and $28 \%$, respectively. Therefore, the restoration of soils with salinity levels upto $24 \mathrm{dS} \mathrm{m}^{-1}$ with these seeds may need some help through addition of particular chemicals that can enhance their salt tolerance before they can germinate naturally. Shahba et al. (2008) studied a saltgrass (Distichlis spicata var. stricta (Greene)) and reported that kinetin and Proxy (at $5 \mathrm{mM}$ ) increased its germination percentage by $54 \%$ under a salinity level of $30 \mathrm{dS} \mathrm{m} \mathrm{m}^{-1}$.

Salinity level of $32 \mathrm{dS} \mathrm{m} \mathrm{m}^{-1}$ is considered as being extremely high where only tolerant species or halophytes can grow (Mishra and Tanna 2017). At this level, the seeds of most species tested in this study had very little or no germination at all (Figure 3). Surprisingly, the seeds of $S$. grandiflora still had a germination of nearly $50 \%$ at this extremely high level of salinity. This could be a potential species which can be used in the future restoration of saltaffected areas. For future work, the establishment stage after germination should be studied in order to test for the survival potential of seedlings and saplings in extremely saline areas. Nichols et al. (2009) studied the salinity tolerance and avoidance mechanisms in five selfregenerating annual pasture legumes of Mediterranean origin during germination. They reported that germination of Melilotus siculus was relatively high at $89 \%$ under $30 \mathrm{dS}$ $\mathrm{m}^{-1} \mathrm{NaCl}$.

Leksungnoen (2017) studied the potential of native Thai species in reclaiming salt-affected areas in Thailand, which included Azima sarmentosa (Salvadoraceae), Gymnosporia mekongensis (Celastraceae), and Buchanania siamensis (Anacardiaceae). The seeds of all the three species could germinate at a salinity level of more than $32 \mathrm{dS} \mathrm{m}^{-1}$, with the total germination ranging between $40-90 \%$. Seeds of these three species are likely to have a better germination percentage when compared to S. Grandiflora, which had a germination of $47 \%$ under $32 \mathrm{dS} \mathrm{m}^{-1} \mathrm{NaCl}$ concentration.

Finally, at a salinity level of $40 \mathrm{dS} \mathrm{m}^{-1}$ (close to the salinity of seawater), which has been reported as the average salinity at the soil surface during an entire year in northeastern Thailand (Leksungnoen 2017), no species could germinate to more than $2 \%$. A. procera, $P$. macrocarpus, and $S$. grandiflora could still germinate to some extent, as indicated by a very low germination percentage (Figure 3). This indicates the difficulty in reclamation through natural means of areas affected by extremely high salinity. This result is similar to the study of germination of three Portuguese beets (Beta vulgaris (, that could only germinate to less than $3 \%$ under salinity of 50 $\mathrm{dS} \mathrm{m}{ }^{-1}$ (Pinheiro et al. 2018).

As the toxicity caused by salinity results in a change in metabolomics processes inside the seed, which leads to a longer germination time (Yildirim et al. 2011; Benjamin et al. 2019). Generally, MGT was longer when salinity levels increased because high salinity results in the lowering of water potential during seed imbibition (Cokkizgin 2012; Aamir et al. 2019). High concentration of $\mathrm{NaCl}$ causes an osmotic barrier and delays the imbibition stage of germination. Many studies have reported that $\mathrm{NaCl}$ can inhibit growth by reducing cell proliferation and cell elongation (Abbasi et al. 2015; Zorb et al. 2015; Valenzuela et al. 2016).

Germination speed describes the number of seeds germinated per day. This provides a more accurate quantification about seedling preparation for planting. It is also known as the efficiency of seeds (Ranal and Santana 2006; Junior et al. 2016). The average germination speed was high under control and gradually decreased as the salinity concentration increased because of low water absorption rate of the seed (Alencar et al. 2015). The germination speed was correlated with MGT, which is consistent with the study of Rosas et al. (2019), who studied the effects of salinity in 2 varieties of lettuce in Brazil. They concluded that salinity resulted in a reduced germination speed because germination percentage decreased. Saengpitak et al. (2018) reported that the 
salinity level had no effect on germination synchrony in the genera from Bambusoideae, which similar to our inference that salinity had no effect on synchrony of the seed.

The highest $\mathrm{EC}_{50}$ value calculated in this study was close to that of Ku-or and Leksungnoen (2018), who reported that Leucaena leucocephala (from the Fabaceae family) had the highest $\mathrm{EC}_{50}$ at $30.86 \mathrm{dS} \mathrm{m}^{-1}$. However, in this study, D. cochinchinensis had a high $\mathrm{EC}_{50}$ value while the total germination percentage was low under all treatments (10-43\%) when compared to $S$. grandiflora and $S$. siamea (Figure 3 ). As can be noted by the formulation of $\mathrm{EC}_{50}$, it is the ratio of germination at salt treatment relative to the germination at control. For D. cochinchinensis, a low germination at control of $31 \%$, when compared to higher salinity levels resulted in a high value of $\mathrm{EC}_{50}$.

Optimal species selection is one of the most important factors when planning for reforestation. For areas affected by salinity, the selected species must be salt tolerant and be able to thrive in saline environments. In addition, socioeconomic considerations would also be important for investors and farmers in terms of the income generated (Budiharta et al. 2016). Our study indicated that seeds of $S$. grandiflora had the highest salt-tolerance among the 16 fabaceous species. It is a medium-sized and fast-growing tree whose young shoots and flowers can be consumed. The bark is also used as a medicine for smallpox (Vipin et al. 2011). In the high EC50 group, S. siamea is a large tree, whose young shoots are consumed by most rural people around Thailand. Its wood can be used as fuel as it has a calorific value of 4,471 cal/g, charcoal obtained from $S$. siamea wood burns efficiently with a calorific value of $6,480 \mathrm{cal} / \mathrm{g}$ (Korang et al. 2015). Similar to that of Rhizophora sp. and Acacia auriculiformis (Hendrati and Nurrohmah 2018). Therefore, this species has several potential benefits for the farmer if considered to be planted in salt-affected areas. D. cochinchinens is a large tree, whose wood is a valuable commodity in Thailand. Its wood is hard and has a distinct texture and is often used for furniture and carving purposes. For this reason alone, $D$. cochinchinensis is in very high demand, especially in China, Singapore, Taiwan, and Hong Kong (Kielmann and Mai 2016; Royal Forest Department 2018). However, illegal logging of this species has continued unimpeded for more than two decades in Thailand. Therefore, if D. cochinchinensis can be established in areas affected by salinity, the lands can be reclaimed with an additional benefit of reduction in illegal logging.

In conclusion, salinity is a continuing problem and has gradually expanded over the years, and so reclamation of salt-affected land is urgently needed. Species that can tolerate salinity are studied to determine the best species that can be used tocope with the problem. The Fabaceae family contains many drought-tolerant species, in which drought responses are somewhat similar to responses to salinity in terms of physiological drought as plant's root has difficulty in extracting water out from a high osmotic soil solution due to high concentration of salinity. Seed germination is an important process during the initial stages of reproduction. It was observed that seed germination generally decreased with an increase in salinity concentration. However, this study showed that most seeds from the 16 Fabaceae species germinated well with germination rates between $30-80 \%$ for salinity concentration up to $24 \mathrm{dS} \mathrm{m}^{-1}$ (considered as very high salt in the soil solution). This indicated that seeds can germinate in most parts afflicted with salinity when the concentration is not extremely high. S. grandiflora, $S$. Siamea, and $D$. cochinchinensis were the most salt-tolerant species found in this study. These three species can be recommended as potential candidates for reclamation of areas affected by salinity. Therefore, further study related to establishment and subsequent growth stages should be investigated to ensure the success of reclamation. Moreover, there might be other species that can tolerate extremely high salinity levels but have yet to be studied or reported. Future screening for salt-tolerant species should be extended to other species in this family and other potential families to have a more diverse portfolio of species for reclamation purposes.

\section{ACKNOWLEDGEMENTS}

This research was supported by the Graduate program scholarship from the Graduate School, Kasetsart University, Bangkok, Thailand.

\section{REFERENCES}

Aamir M, Khan EA, Baloch MS, Aslam M. 2019. Germination ecology of Lathyrus aphaca, a prolematic weed of wheat crop under semi-arid conditions of Pakistan. Planta Daninha 37:1-10. DOI: 10.1590/s010083582019370100048

Abbasi GH, Akhtar J, Anwar-ul-Haq M, Malik W, Ali S, Chen ZH, Zhang G. 2015. Morpho-physiological and micrographic characterization of maize hybrids under $\mathrm{NaCl}$ and $\mathrm{Cd}$ stress. Plant Growth Regulation 75 (1): 115-122.

Ahmad P, Azooz MM, Prasad MNV. 2013. Salt Stress in Plants: Signalling, Omics and Adaptations. Springer Science+Business Media, New York

Alencar NL, Gadelha CG, Gallão MI, Dolder MA, Prisco JT, GomesFilho E. 2015. Ultrastructural and biochemical changes induced by salt stress in Jatropha curcas seeds during germination and seedling development. Funct Plant Biol 42 (9): 865-874.

Aravind J, Vimala DS, Radhamani J, Jacob SR, Kalyani S. 2019. The germinationmetrics Package: A Brief Introduction. ICAR-National Bureau of Plant Genetic Resources, Mew Delhi.

Arunin S. 1989. Northeast Saline Soil. Department of Land Development, Bangkok. [Thailand]

Arunin S. 1996. Soil Salinity in Thailand. Department of Land Development, Ministry of Agriculture and Cooperatives, Bangkok. [Thailand]

Azani N, Babineau M, Bailey CD et al. 2017. A new subfamily classification of the Leguminosae based on a taxonomically comprehensive phylogeny. Taxon 66 (1): 44-77.

Baskin CC, Baskin JM. 2014. Seeds: Ecology, biogeography and evolution of dormancy and germination, 2nd ed. Elsevier/Academic Press, San Diego, CA.

Benjamin JJ, Lucini L, Jothiramshekar S, Parida A. 2019. Metabolomic insights into the mechanisms underlying tolerance to salinity in different halophytes. Plant Physiol Biochem 135: 528-545.

Bewley JD, Black M. 2012. Physiology and Biochemistry of Seeds in Relation to Germination: Volume 2: Viability, Dormancy, and Environmental Control. Springer Science \& Business Media, New York. 
Budiharta S, Meijaard E, Wells JA, Abram NK, Wilson KA. 2016. Enhancing feasibility: Incorporating a socio-ecological system framework into restoration planning. Environ Sci Policy 64: 83-92.

Bueno M, Lendínez ML, Aparicio C, Cordovilla MP. 2017. Germination and growth of Atriplex prostrata and Plantago coronopus: Two strategies to survive in saline habitats. Flora 227: 56-63.

Chanrattana P, Channa P, Sovanna P, Gray TNE. 2013. Structure and composition of deciduous dipterocarp forest in the Eastern Plains Landscape, Cambodia. Cambodian J Nat Hist 1: 27-34.

Chikoto H, Eloff JN, Swan GE, Bizimenyera SE. 2005. Rationale for using Peltophorum africanum (Fabaceae) extracts in veterinary medicine. J S Af Vet Assoc 76: 54-58.

Cokkizgin A. 2012. Salinity stress in common bean (Phaseolus vulgaris L.) seed germination. Not Bot Horti Agrobo 40: 177-182.

Egamberdieva D, Mamedov NA. 2015. Potential use of Licorice in phytoremediation of salt affected soils. In Plants, Pollutants and Remediation. Springer, Dordrecht.

Gunes F, Ali C. 2011. Seed characteristics and testa textures some taxa of genus Lathyrus L. (Fabaceae) from Turkey. Int J Agric Biol 13: 888894.

Han C, Yang P. 2015. Studies on the molecular mechanisms of seed germination. Proteomics 10 (15): 1671-1679.

Hendrati RL, Nurrohmah SH. 2018. Quality of Genetically-Improved Acacia auriculiformis For Renewable Short-Rotation Wood-Energy. Jurnal Manajemen Hutan Tropika 24 (3): 136-143.

Hubbard M, Germida J, Vujanovic V. 2012. Fungal endophytes improve wheat seed germination under heat and drought stress. Botany 90 137-49. DOI: 10.1139/b11-091.

ISTA. 2017. International Rules for Seed Testing: Rule1999. Internationa Seed Testing Association, Zurichstr, Switzerland.

Izadi MH, Rabbani J, Emam Y, Pessarakli M, Tahmasebi A. 2014. Effects of salinity stress on physiological performance of various wheat and barley cultivars. J Plant Nutr 37: 520-531.

Jaganathan GK, Wu GR, Han YY, Liu BL. 2017. Role of the lens in controlling physical dormancy break and germination of Delonix regia (Fabaceae: Caesalpinioideae). Plant Biol 19 (1): 53-60.

Jayasuriya KG, Wijetunga AS, Baskin JM, Baskin CC. 2010. Recalcitrancy and a new kind of epicotyl dormancy in seeds of the understory tropical rainforest tree Humboldtia laurifolia (Fabaceae, Ceasalpinioideae). Am J Bot 97 (1): 15-26.

Jayasuriya KMGG, Wijetunga ASTB, Baskin JM, Baskin CC. 2013. Seed dormancy and storage behaviour in tropical Fabaceae: A study of 100 species from Sri Lanka. Seed Sci Res 23: 257-269.

Jedrum S, Thanachit S, Anusontpornperm S, Wiriyakitnateekul W. 2014 Soil amendments effect on yield and quality of jasmine rice grown on typic natraqualfs, Northeast Thailand. Intl J Soil Sci 9 (2): 37-54.

Junior CA, de Oliveira Vitoriano J, Da Silva DLS, de Lima Farias M, de Lima Dantas NB. 2016. Water uptake mechanism and germination of Erythrina velutina seeds treated with atmospheric plasma. Sci Rep 6 : 33722. DOI: $10.1038 /$ srep33722.

Kheoruenromne I. 2007. Saline soil in Thailand. Publisher Kasetsart University, Bangkok. [Thailand]

Kielmann BC, Mai C. 2016. Natural weathering performance and the effect of light stabilizers in water-based coating formulations on resin-modified and dye-stained beech-wood. J Coatings Technol Res 13 (6): 1065-1074. DOI: 10.1007/s11998-016-9818-0.

Korang JK, Obiri BD, Appiah H, Awuku S. 2015. Calorific values and gravimetric yield of six wood fuel species in the forest transition zone of Ghana. CSIR-Forestry Research Institute of Ghana 31: 51-61.

$\mathrm{Ku}$-or Y, Leksungnoen N. 2018. Germination comparison of eight exotic and native forest tree species at different salt concentration levels. Thai J For 37: 1-12. [Thailand]

Leksungnoen N, Kjelgren KR, Beeson C, Richard JR, Paul GJ, Grant EC, Austin H. 2014. Salt tolerance of three tree species differing in native habitats and leaf traits. Hortscience 49: 1194-1200.

Leksungnoen N. 2017. Reclaming saline areas in Khorat Basin Northeast Thailand: Soil properties, species distribution, and germination of potential tolerant species. Arid Land Res Manag 31: 235-252.

Linkies A, Graeber K, Knight C, Leubner-Metzger G. 2010. The evolution of seeds. New Phytologist 186: 817-831. DOI:10.1111/j.14698137.2010.03249.x

Long RL, Wilkliams K, Griffiths EM, et al. 2010. Prior hydration of Brassica tournefortii seeds reduces the stimulatory effect of karrikinolide on germination and increases seed sensitivity to abscisic acid. Ann Bot 105: 1063-1070.
Metternicht G, Zinck A. 2008. Remote Sensing of Soil Salinization. CRC Press, Boca Raton. DOI: 10.1201/9781420065039.

Mishra A, Tanna B. 2017. Halophytes: Potential resources for salt stress tolerance genes and promoters. Frontiers Plant Sci 8: 829. DOI: $10.3389 /$ fpls.2017.00829.

Moud AM, Maghsoudi K. 2008. Salt stress effects on respiration and growth of germinated seeds of different wheat (Triticum aestivum L.) cultivars. World J Agric Sci 4: 351-358.

Munns R. 2002. Comparative physiology of salt and water stress. Plant Cell Environ 25: 239-350. DOI: 10.1046/j.0016-8025.2001.00808.x.

Munns R, Tester M. 2008. Mechanisms of salinity tolerance. Ann Rev Plant Biol 59: 651-681

Nawaz A, Amjad M, Jahangir MM, Khan SM, Cui H, Hu J. 2012. Induction of salt tolerance in tomato (Lycopersicon esculentum Mill.) seeds through sand priming. Aust J Crop Sci 6 (7): 1199-1203.

Nichols PGH, Malik AI, Stockdale M, Colmer TD. 2009. Salt tolerance and avoidance mechanisms at germination of annual pasture legumes: Importance for adaptation to saline environments. Plant Soil 315: 241-255. DOI: 10.1007/s11104-008-9747-5.

Pakar N, Pirasteh-Anosheh H, Emam Y, Pessarakli M. 2016. Barley growth, yield, antioxidant enzymes and ions accumulation affected by PGRs under salinity stress. J Plant Nutr 39 (10): 1372-1379.

Panuccio MR, Jacobsen SE, Akhtar SS, Muscolo A. 2014. Effect of saline water on seed germination and early seedling growth of the halophyte quinoa. AoB Plants Open Access J Plant Sci 6 (47): 1-18.

Perez-Harguindeguy N, Diaz S, Garnier E et al. 2013. New handbook for standardized measurement of plant functional traits worldwide. Australian J Bot 61: 167-234. DOI: 10.10 71/BT12225

Pessarakli M. 2015. Effects of a bio-stimulant and salinity stress on growth and quality of ryegrass (Lolium prenne L.), an urban desert landscape and forage crop, for sustainable agriculture in arid regions. Intl J Water Resour Arid Environ 4 (2): 94-104.

Pinheiro C, Ribeiro IC, Reisinger V, Planchon S, Veloso MM, Renaut J, Eichacker L, Ricardo CP. 2018. Salinity effect on germination, seedling growth and cotyledon membrane complexes of a Portuguese salt marsh wild beet ecotype. Theor Exp Plant Physiol 30: 113. DOI: 10.1007/s40626-018-0107-4.

Ranal MA, Santana DG. 2006. How and why to measure the germination process?. Rev Bras Bot 29 (1): 1-11.

Rosas JTF, Junior EM, Lorenzoni RM, Santos FFLD, Martins RN. 2019. Effect of salinity on germination of lettuce cultivars produced in Brazil. J Exp Agric Int 34: 1-8.DOI: 10.9734/JEAI/2019/v34i530183.

Royal Forest Department. 2018. A Guide to Sustainable Economic Forest Plantation Management. Department of Forest Certification, Royal Forest Department, Bangkok. [Thailand]

Saengpitak S, Leksungnoen N, Sungkaew S. 2018. Germination of bamboo seeds under salt stress. In National Proceeding Thaksin University: Research and Innovation for Social Stability, Prosperity and Sustainability, Bangkok. [Thailand]

Schmidt L. 2007. Tropical Forest Seed. Springer-Verlag, Berlin.

Shahba MA, Qian YL, Lair KD. 2008. Improving seed germination of saltgrass under saline conditions. Crop Sci 48: 756-762. DOI: 10.2135/cropsci2007.07.0382.

Srivastava S, Kumar K, Srivastava M. 2012. Effect of different presowing treatments on seed germination of Cassia occidentalis. Res Environ Life Sci 5: 153-155.

Santisuk T. 2006. Forest of Thailand. Forest Herbarium, Department of National Park, Wildlife and Plant Conservation, Bangkok. [Thailand]

Todaria NP, Negim AK. 1992. Pretreatment of some Indian Cassia seeds to improve their germination. Seed Sci Technol 20: 583-588.

US Salinity Laboratory Staff. 1954. Diagnosis and improvement of saline and alkali soils. USDA Handbook 60. U.S. Government Printing Office, Washington, DC.

Valenzuela CE, Acevedo-Acevedo O, Miranda GS, Vergara-Barros P, Holuigue L, Figueroa CR, Figueroa PM. 2016. Salt stress response triggers activation of the jasmonate signaling pathway leading to inhibition of cell elongation in Arabidopsis primary root. J Exp Bot 64 (14): 4209-4220.

Vipin K, Arun KG, Rajesh G. 2011. Pharmacognostical investigation on Sesbania grandiflora (L.) pers. Intl J.Pharm Sci Res 2: 1069-1072.

Vozzo JA. 2002. The tropical tree seed manual. U.S. Department of Agriculture, Forest Service. U.S. Government Printing Office, Washington, DC:

Weitbrecht K, Muller K, Leubner-Metzger G. 2011. First off the mark: Early seed germination. J Exp Bot 62: 3289-3309. 
Yahara T, Javadi F, Onoda Y, et al 2013. Global legume diversity assessment: Concepts, key indicators, and strategies. Taxon 62: 249266. DOI: $10.12705 / 622.12$

Yildirim E, Karlidag H, Dursun A. 2011. Salt tolerance of Physalis during germination and seedling growth. Pak J Bot 43: 2673-2676.
Zorb C, Muhling KH, Kutschera U, Geilfus CM. 2015. Salinity stiffens the epidermal cell walls of salt-stressed maize leaves: is the epidermis growth-restricting?. PLoS One 10 (3). e0118406. DOI: 10.1371/journal.pone.0118406. 